\title{
PB 9493-9494 intertacting galaxies: The correlation of IRAS sources with close pairs of PB UVe objects ${ }^{\star}$
}

\author{
H. Reboul ${ }^{1}$ and C. Vanderriest ${ }^{2}{ }^{\star \star \star}$ \\ ${ }^{1}$ UMR 5024, CNRS-Université Montpellier 2, GRAAL, CC 72, 34095 Montpellier Cedex 5, France \\ 2 UMR 8631, CNRS-DAEC, Observatoire de Paris, 92195 Meudon, France \\ Received 12 September 2000 / Accepted 22 August 2002

\begin{abstract}
The cross-correlation of close pairs of UV-excess objects from the Berger-Fringant catalogue with IRAS sources supplies three cases. The first two are already known objects. We study in detail the most interesting (and new) case: PB 9493-9494. Optical observations in direct imaging and in integral field spectrography confirm that this system is a pair of strongly inter-
\end{abstract} \\ acting galaxies with starbursts and show the complex structure of an evolved but not merged encounter. Although such an \\ evolution step is only intermediate, the brightest nucleus (PB 9494) may be on the verge of becoming an AGN. We discuss the \\ peculiarities of this system with regard to optical versus far infrared behaviour, in connection with its special mode of selection.
}

Key words. galaxies: individual: PB 9494, PB 9493 - galaxies: interactions - galaxies: starburst - ultraviolet: galaxies infrared: galaxies

\section{Introduction}

Observations and models have gradually shown that galaxy interactions and merging are a key mechanism for triggering bright nuclear starbursts as well as AGNs. The process is now well documented for starburst galaxies (Halpern et al. 1984; Shaver \& Chen 1985). For quasars, the first serious clues came from a comprehensive study of a low-redshift sample (Stockton $\&$ MacKenty 1987), and many further theoretical and observational studies confirm that the process is valid for the brightest objects too (see e.g. Djorgovski et al. 1987; Crampton et al. 1988; Bahcall et al. 1997). It is also recognized that such processes are the bright sides or episodes of a more general phenomenon among galaxies: evolution through binary or multiple encounters.

We have already proposed (Reboul et al. 1985) to name "interactivation" the whole set of dissipative processes by which a close encounter of galaxies triggers massive nuclear starbursts and (sometimes) true active nuclei that are rich in cosmological developments (Reboul \& Cordoni 2002). The evolution that turns nuclear starbursts into active nuclei is still not perfectly understood but it is likely (from the relative frequencies and durations of the two classes of phenomena) that the occurence of this transformation needs some additional conditions.

We found that an efficient way to identify "interactivating" galaxies is to search for close pairs of UV-excess (hereafter UVe) objects (Reboul et al. 1987; Vanderriest \& Reboul 1991).

Send offprint requests to: $\mathrm{H}$. Reboul,

e-mail: reboul@graal .univ-montp2.fr

* Based on observations collected with the Canada-France-Hawaii Telescope at Mauna Kea (Hawaii, USA).

$\star \star$ Deceased 12 February 2002.
This is one of the reasons why we are completing a systematic observation of all the close pairs in available wide field catalogues of faint UVe objects mainly in the Berger Fringant (hereafter BF) catalogue (Berger \& Fringant 1977, 1980, 1984) which covers 1400 square degrees with Schmidt plates at a limiting $B$-mag 19.5. An automated and more general-purpose survey with other Schmidt plates and the MAMA microdensitometer (Berger et al. 1991; Moreau \& Reboul 1995) is currently in progress in this direction.

Another well-known rich source of such interactivating galaxies is the IRAS whole sky survey. However, the redshift limit for galaxies in the IRAS catalogue is close to $z_{\mathrm{c}}=0.04$ : this is shown for general IRAS galaxies by the sample investigations of Vader \& Simon (1987, their Fig. 2) which is also true - save for a softer cut-off in the distribution of redshifts - for samples of MacAlpine emission-line galaxies (Salzer \& MacAlpine 1988, their Fig. 10). Luminous Infra-red Galaxies show systematic signs of binary interactions (Murphy et al. 1996). At higher reshifts, this process of binary encounters and merging is also an important factor in galaxy evolution. It could noticeably contribute to the IR background (see Wang \& Biermann 2000 and references therein). Our previous detailed investigations of close pairs of PB faint UVe "interactivated" galaxies (Vanderriest \& Reboul, 1991) concerned sources with $z=0.06$ to 0.09 whose absence in the IRAS catalogue was not surprising.

It is generally assumed that most of the mid-far infrared $(10-50 \mu \mathrm{m})$ flux of "interactivating" galaxies is due to the thermal re-emission of dust heated mainly by the UV radiation of young massive OB stars in the burst (without excluding the contribution of a non-thermal enshrouded true AGN for the central parts). This implies that a sufficient amount of dust is 
close to the ionizing sources. For the true far infrared (FIR) part of the spectrum (including 60 and $100 \mu \mathrm{m}$ IRAS bands), the emitting dust may significantly be heated by non-ionizing photons. Although the correlation between UVe and IR excess (IRe) does not seem questionable, it may be hidden if a large amount of dust absorbs most of the direct UV radiation or reduced if the amount of dust close to the young UV stars is still too low. The constitution of complete samples of active galaxies has then to deal with the complementarity of UVe and IRe selection techniques (Salzer \& MacAlpine 1988; Mazzarella et al. 1991).

Our a priori selection of close pairs of resolved UVe objects specifically combines binarity with UVe. Compared to the IRAS survey, we go deeper in redshift and favour the detection of "interactivating" galaxies. The complete PB catalogue of 9010 faint UVe objects contains 8 pairs separated by no more than $10^{\prime \prime}$, plus 3 one-entry objects noted as having an UVe companion in the same range of angular separation. Among them, three systems are present in the IRAS faint source catalogue, version 2 . They have, respectively, the following $B$-magnitudes (and colour classes) in the BF catalogue:

- PB 3933-PB 3934: 17.5 (I), 17.5 (I)

- PB 6008-PB 6009: 16 (II), 18 (II)

- PB 9493-PB 9494: 16 (II), 15 (I).

PB 6008, PB 9493 and PB 9494 are referred as "compact object" by BF.

The components of the first pair obviously are (as noted by BF) two HII regions in NGC 4254 (also M99 and IRAS 12162+1441). They lie in an arm and then (for $z=$ 0.008 ) have absolute magnitudes $M_{B} \sim-15$.

The second pair is (as noted by BF) the Mac Alpine galaxy UM 254. This object has been widely observed (see e.g. Mac Alpine et al. 1977; Thomson \& Djorgovski 1990; Terlevich et al. 1991). Its morphology is quite simple (Keel 1985). The brighest component, PB 6008, is a Sy2 galaxy at $z=0.0444$ and has $J, H$ and $K$ magnitudes in the 2MASS catalogue (respevctively 14.9, 14.4 and 13.8). The simple extension, PB 6009, is not catalogued in 2MASS where it appears at least 2 magnitudes fainter on the maps.

The third pair, not previously studied, clearly seemed the most interesting one. Its angular separation, its UVe, the "compactness" of both its components and their close $B$ magnitudes characterized a high grade candidate for "interactivation". A preliminary picture by P. Magain with the ESO $2.2 \mathrm{~m}$ telescope in November 1986 confirmed this possibility.

By chance, this unique UVe-IRe close pair of "compact" PB objects falls (without cross-identification) into the 140 square degree sample of 92 IRAS galaxies investigated by Vader \& Simon (1987) where they are noted as an (uncatalogued) double object with " $J$ " $\left(B_{J}\right)$ magnitudes 15.62 and 16.34 and galactocentric radial velocities of 8886 and $8930 \mathrm{~km} \mathrm{~s}^{-1}$.
Table 1. Journal of observations.

\begin{tabular}{lrcccc}
\hline \hline mode & date & $\begin{array}{c}\text { expos. } \\
(\mathrm{mn})\end{array}$ & $\begin{array}{c}\text { wavelength } \\
\text { range }(\AA)\end{array}$ & $\begin{array}{c}\text { dispers. } \\
(\AA / \mathrm{mm})\end{array}$ & $\begin{array}{c}\text { sampling } \\
\left({ }^{\prime \prime}\right)\end{array}$ \\
\hline image & 88.12 .04 & 10 & $R$ filter & & 0.23 \\
image & 88.12 .04 & 10 & $V$ filter & & 0.23 \\
IFS & 88.12 .03 & 10 & $4300-7000$ & 235 & 1.4 \\
IFS & 88.12 .03 & 15 & $4700-6000$ & 100 & 1.4 \\
IFS & 88.12 .03 & 15 & $6000-7300$ & 100 & 1.4 \\
IFS & 88.12 .04 & 20 & $4300-7000$ & 235 & 0.7 \\
\hline
\end{tabular}

\section{Observations and data analysis of PB 9493-9494 (= IRAS 03051-1206)}

PB 9493 and PB 9494 have been observed with the SILFID spectrograph (Vanderriest \& Lemonnier 1988) at the Cassegrain focus of the $3.6 \mathrm{~m} \mathrm{CFH}$ telescope.

\subsection{Observation material}

The journal of observations is given in table 1 . In imaging mode, the spatial sampling of the CCD was $20 \mu \mathrm{m}\left(0.23^{\prime \prime}\right)$. In spectrographic mode (integral field spectrography, hereafter IFS), the spatial sampling in the focal plane is fixed by the size of the $100 \mu \mathrm{m}$ fibres. Each fibre represents $0.7^{\prime \prime}$ in the direct mode $(\mathrm{F} / 8)$ and 1.4" with an F/4 focal reducer. For all these observations, the seeing was about $0.8^{\prime \prime}$. Because of the small size of the CCD $(512 \times 512$ pixels $)$ at the time of observation, only about $1 / 3$ of the IFS field of view can be recorded. We aligned the elongated window along the PB 9493-9494 axis (Fig. 1).

\subsection{Data reduction}

The data reduction procedures for the IFS mode of SILFID are fully described elsewhere (Vanderriest 1995). The software package allows us to extract and calibrate individual fibre spectra and automatically reconstitute images in flux (either continuum or line), maps of line redshift (velocity), line $F W H M$, or any combination of those parameters. This allows us to define physical regions in which spectra of individual fibres can be safely co-added.

\section{Results}

\section{1. morphology}

The general morphology on the direct imaging picture (Fig. 1) is suggestive of a strong tidal interaction. The brightest galaxy PB 9494 shows at least 5 distinct knots within $2.5^{\prime \prime}$ and 2 main extensions looking like antennæ. The core of the companion PB 9493 looks slightly fuzzier and is surrounded by irregular patches. These two bodies are linked by a faint curved bridge and are embedded in a common low surface brightness envelope extending more than $25^{\prime \prime}$. 


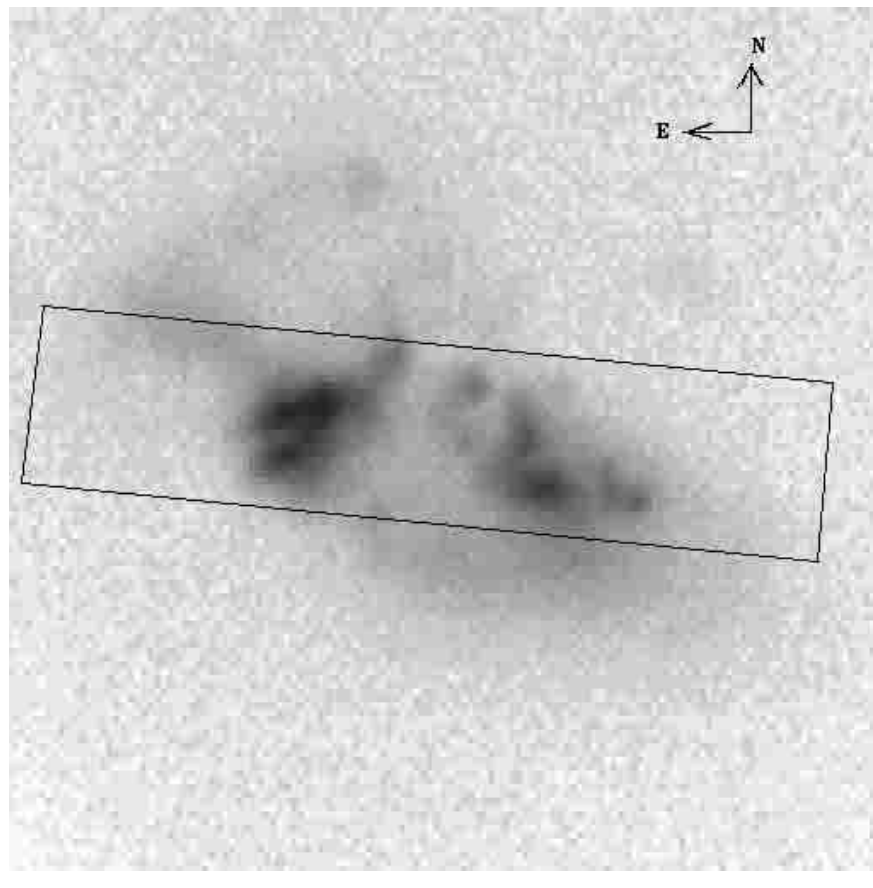

Fig. 1. CCD picture of PB 9493-9494 with $R$-filter (logarithmic gray scale, PB 9494 is at left). The field is $\sim 30 \times 30$ arcsec, the seing $\sim 0.8$ arcsec. The rectangular box shows the area covered in IFS (see Figs. 2 and 4).

\section{2. integral field spectrophotometry}

The different components identified by direct imaging are also easily recognizable in the pictures reconstructed from the IFS data (taking into account the difference of spatial sampling). Figure 2 displays the reconstructed field in the continuum and [O III] emission lines. One can see that the tidal feature labeled E1 is mostly prominent in continuum while regions E2 and E3 are visible on both maps. Also, it is obvious that the brightness ratio between the 2 galaxies is higher in gas emission than in stellar light. This can be measured on the integrated (coadded) spectra (Fig. 3) of the nuclear regions (hereafter PB 9493N and PB 9494N).

Both spectra show blue continua without significant absorption lines but with unresolved $\left(F W H M<200 \mathrm{~km} \mathrm{~s}^{-1}\right)$ strong emission lines.

Table 2 summarizes the main information extracted from our bi-dimensional spectrography of the whole system. The spectra have been calibrated by use of star HZ 14 (Oke 1974).

\section{3. reddening}

It is necessary to know the extinction for a discussion of the excitation mechanism(s). The relatively low intensities of $\left[\mathrm{O}_{\mathrm{I}}\right]_{6300}$ and of the $\left[\mathrm{S}_{\mathrm{II}}\right]$ doublet indicates that a contribution by shock excitation may exist but should be moderate. In a first approximation, we can neglect it. With the assumption of a pure radiative process, we can use the Balmer decrement for measuring the reddening. Intrinsic intensities $I(\lambda)$ are deduced from the observed intensities $F(\lambda)$ by:

$I(\lambda)=F(\lambda) 10^{c f(\lambda)}$,
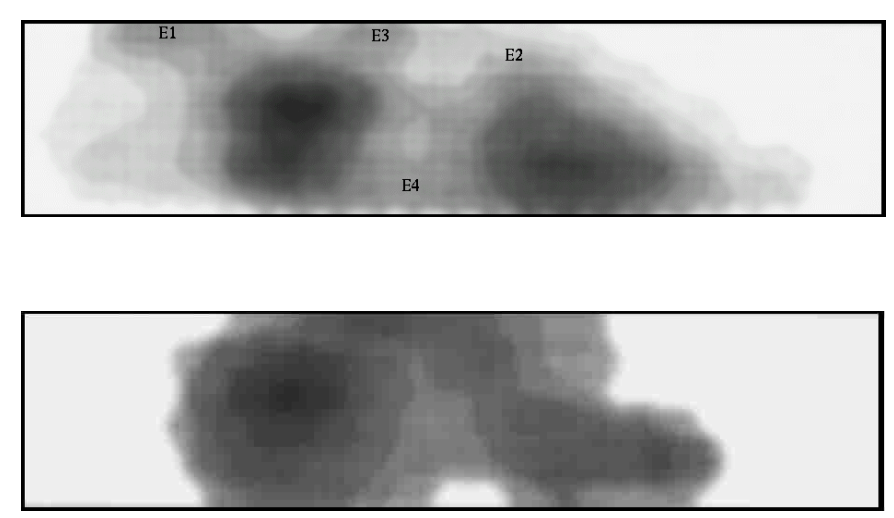

Fig. 2. Reconstructed pictures (shown in logarithmic gray scale) from IFS data. Upper image: in the continuum light (5200-5900 ̊), with identification of the (tidal ?) extensions. Lower image: in the $\left[\mathrm{O}_{\text {III }}\right]_{5007}$ emission line.
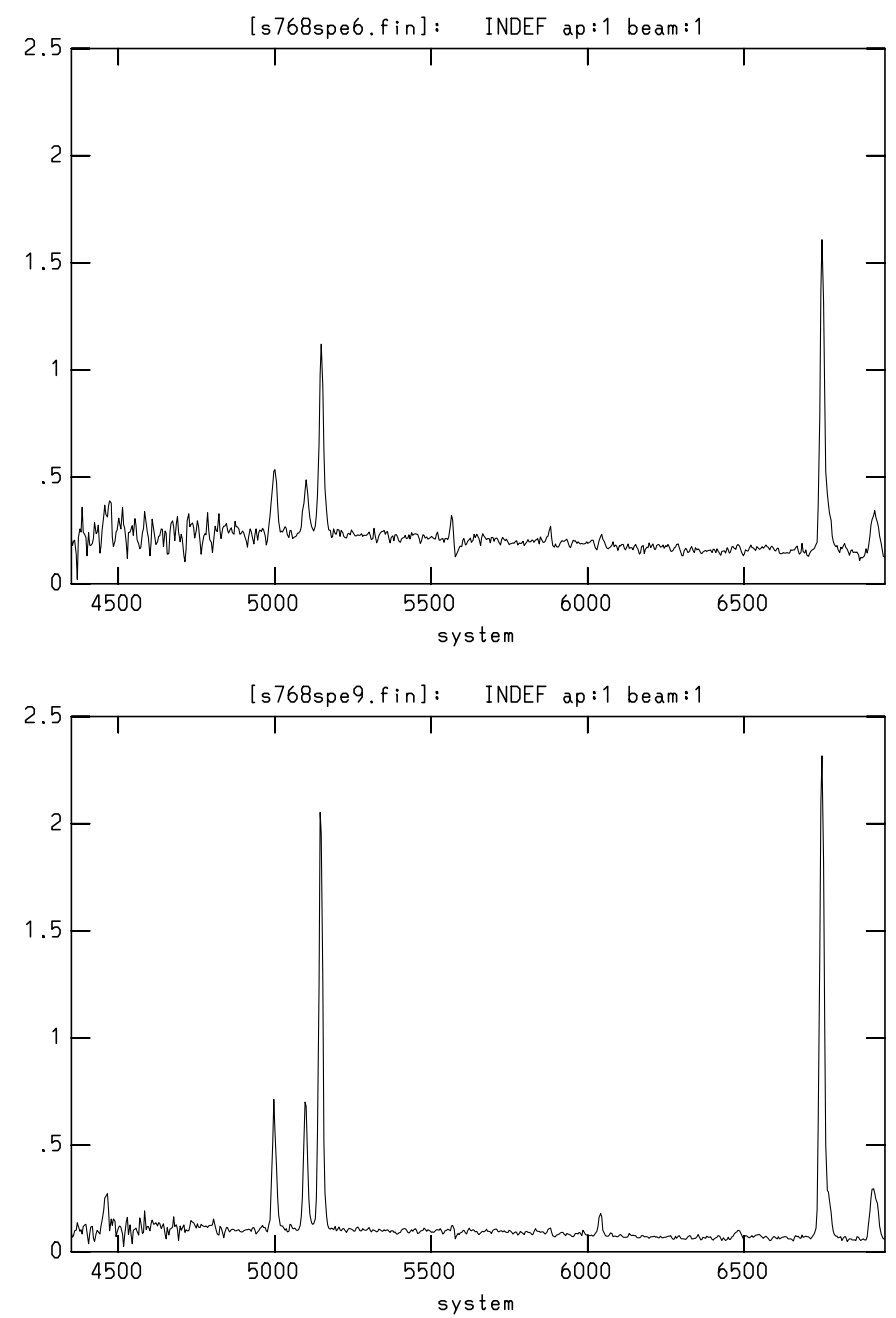

Fig. 3. Integrated spectra of the central starbursts. Upper image: region PB 9493N; lower image: region PB 9494N (brightest knot).

where $f(\lambda)$ is the standard Whitford reddening curve (Whitford 1958) as parametrized by Miller \& Matthews (1972) and $c$ a constant that depends on $E(B-V)$. From the maps given by Burstein \& Heiles (1982) and the galactic coordinates 
Table 2. Relative line fluxes.

\begin{tabular}{|c|c|c|c|c|c|}
\hline zone $\rightarrow$ & PB 9493N & PB 9493 & PB 9494N & PB 9494 & $E 2$ \\
\hline Area $\rightarrow$ & $5.3^{\prime \prime 2}$ & $30^{\prime \prime 2}$ & $1.3^{\prime \prime 2}$ & $36^{\prime \prime 2}$ & $4^{\prime \prime 2}$ \\
\hline \multicolumn{6}{|l|}{ line $\downarrow$} \\
\hline $\mathrm{H}_{\beta}$ & 100 & 100 & 100 & 100 & 100 \\
\hline$[\mathrm{O} \text { III }]_{4959}$ & $67 \pm 13$ & $71 \pm 12$ & $97 \pm 12$ & $91 \pm 8$ & $95 \pm 25$ \\
\hline$[\mathrm{O} \text { III }]_{5007}$ & $231 \pm 25$ & $219 \pm 20$ & $328 \pm 25$ & $296 \pm 15$ & $268 \pm 50$ \\
\hline $\mathrm{He}_{5} 5876$ & & & $23 \pm 4$ & $11 \pm 2$ & \\
\hline$[\mathrm{O} \text { I }]_{6300}$ & & & $25 \pm 4$ & $13 \pm 3$ & \\
\hline$\left[\mathrm{N} \mathrm{II}_{6548}\right.$ & $12 \pm 4$ & $20 \pm 5$ & $8 \pm 3$ & $6 \pm 2$ & \\
\hline $\mathrm{H}_{\alpha}$ & $376 \pm 30$ & $382 \pm 25$ & $388 \pm 25$ & $347 \pm 20$ & $257 \pm 50$ \\
\hline$\left[\mathrm{N}_{\text {III }}\right]_{6583}$ & $34 \pm 6$ & $37 \pm 6$ & $34 \pm 5$ & $28 \pm 4$ & $14 \pm 5$ \\
\hline$\left[\mathrm{S}_{\text {III }}\right]_{6717}$ & $52 \pm 7$ & $51 \pm 7$ & $39 \pm 5$ & $38 \pm 4$ & $24 \pm 7$ \\
\hline$\left[\mathrm{S}_{\mathrm{II}}\right]_{6731}$ & $34 \pm 6$ & $49 \pm 7$ & $25 \pm 4$ & $25 \pm 4$ & $22 \pm 7$ \\
\hline $\begin{array}{l}<\mathrm{cz}>\rightarrow \\
\left(\mathrm{km} \mathrm{s}^{-1}\right)\end{array}$ & $8965 \pm 20$ & $8954 \pm 20$ & $8874 \pm 20$ & $8895 \pm 2$ & $9000 \pm 35$ \\
\hline
\end{tabular}

Table 3. Absolute line fluxes and (derreddened) intensities in units $10^{-18} \mathrm{~W} \mathrm{~m}^{-2}$.

\begin{tabular}{lcccc}
\hline \hline zone & $f\left(\left[\mathrm{O}_{\mathrm{III}}\right]_{5007}\right)$ & $f\left(\mathrm{H}_{\alpha}\right)$ & $I\left(\left[\mathrm{O}_{\mathrm{III}}\right]_{5007}\right)$ & $I\left(\mathrm{H}_{\alpha}\right)$ \\
\hline PB 9493N & 13.5 & 22. & $30 .(15.5)$. & $38 .(24)$. \\
PB 9493 & 39. & 68. & $91 .(45)$. & $122 .(75)$. \\
PB 9494N & 24. & 25. & $51 .(28)$. & $46 .(27.5)$. \\
PB 9494 & 197. & 231. & $348 .(227)$. & $343 .(255)$. \\
E1 & 0.6 & 1. & & \\
E2 & 10. & 9.5 & & \\
E3 & 4.8 & 10.3 & & \\
E4 & $<0.5$. & $<0.5$ & & \\
\hline
\end{tabular}

$\left(l=194.2^{\circ}, b=-54.7^{\circ}\right)$, we can estimate the galactic reddening toward PB 9493-9494: $E(B-V)=0.045 \pm 0.01$ which gives an extinction $A_{V} \approx 3 E(B-V)=0.135$.

The measured ratio of the $\left[\mathrm{S}_{\mathrm{II}}\right]$ doublet ensures that the electron density is not significantly larger than $N_{\mathrm{e}}=100 \mathrm{~cm}^{-3}$; the $[\mathrm{O} \text { III }]_{4363}$ line is not easily measurable, it lies in a noisy portion of the spectrum. We will assume that $T_{\mathrm{e}}=10^{4} \mathrm{~K}$. With these parameters, the theoretical $\mathrm{H}_{\alpha} / \mathrm{H}_{\beta}$ ratio should be around 2.85, while we measure larger values. For instance 3.88 for PB $9494 \mathrm{~N}$ indicates a total extinction at $\mathrm{H}_{\beta}: \mathrm{A}_{\mathrm{H} \beta}=0.90$ mag (from which 0.16 mag is due to the Milky Way) for that nuclear region. Similar de-reddenning corrections have been applied to the other parts.

Table 3 displays fluxes and reddening-corrected intensities of the main regions (values between parentheses are corrected for Milky Way reddening only). From the emission lines, we obtain a mean heliocentric redshift $\langle z>=0.02982 \pm$ $0.00002(0.02971 \pm 0.00002$ in galactocentric frame $)$ with a small velocity dispersion between the different regions. The arms have no significant radial velocities relative to the galaxies but a significant structure is seen in the velocity field near the condensations of PB 9494 (Fig. 4). An extremum of radial velocity lies at $1.75 h_{50}^{-1} \mathrm{kpc}$ (projected separation) of its nucleus with $v_{r} \sim-40 \mathrm{~km} \mathrm{~s}^{-1}$ relatively to the mean redshift.

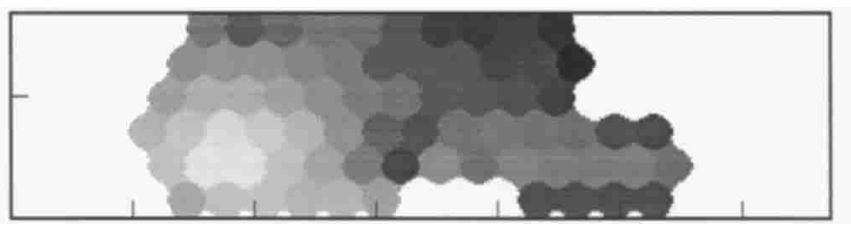

Fig. 4. Velocity field in [O $\mathrm{III}]$ displayed in gray scale (white for $c z \simeq$ $8700 \mathrm{~km} \mathrm{~s}^{-1}$, black for $c z \simeq 9000 \mathrm{~km} \mathrm{~s}^{-1}$ ).

\section{Discussion}

\subsection{Infra-red data}

\subsubsection{IRAS}

The flux densities measured by IRAS for this (unresolved) system are (in Jy for each IR wave-band): $<.25(12 \mu \mathrm{m}),<.32(25 \mu \mathrm{m}), .80 \pm .08(60 \mu \mathrm{m})$ and $1.25 \pm$ $.10(100 \mu \mathrm{m})$. Spectral indices $\alpha$ (defined as usual with $F_{v} \propto$ $v^{\alpha}$ ) are then $\alpha_{25}^{60}<-1$. and $\alpha_{60}^{100}=-0.9$. An $f_{60} / f_{100}$ ratio equal to 0.64 corresponds to a starburst (Sekiguchi 1987) with a warm FIR colour temperature, which suggests that the bulk of the emitting dust is close to the exciting stars and that the starburst is rather young.

The total far infrared $(40-500 \mu \mathrm{m})$ flux $F_{\text {FIR }}$ may be estimated from the flux densities at 60 and $100 \mu \mathrm{m}$ with the procedure of Lonsdale (1985) quoted by Coziol (1996): $F_{\mathrm{FIR}}=$ $1.26 \times 10^{-14}\left(2.58 f_{60}(\mathrm{Jy})+f_{100}(\mathrm{Jy})\right) \mathrm{W} \mathrm{m}^{-2}$. For the PB system, $F_{\text {FIR }}=4.2 \times 10^{-14} \mathrm{~W} \mathrm{~m}^{-2}$.

Assuming a spectral index $\alpha \sim-1$ (close to the IRAS value) the FIR luminosity may then be related to the flux by:

$$
L_{\mathrm{FIR}} \sim F_{\mathrm{FIR}} \times 4 \pi\left(c z / H_{\circ}\right)^{2}(1+z)^{2}
$$

a relation (valid for low redshifts) equivalent to that of Lonsdale (ibidem). So, if the $F_{\text {FIR }}$ evaluation procedure above stands for our PB system, its FIR luminosity would be $L_{\mathrm{FIR}}=1.7 \times$ $10^{37} h_{50}^{-2} \mathrm{~W}$ or $4.5 \times 10^{10} h_{50}^{-2} L_{\odot}$. Ultra-luminous IR galaxies (ULIRG) are defined, more or less arbitrarily, as having $L_{\mathrm{FIR}}>10^{12} L_{\odot}$ and luminous IR galaxies as $L_{\mathrm{FIR}}>10^{11} L_{\odot}$. The PB 9493-9494 system is thus very close to the latter lower limit. In this range of FIR luminosity, $\sim 10 \%$ of galaxies are strongly interacting (Sanders et al. 1988).

\subsubsection{MASS}

On 2MASS maps (Fig. 5), the morphology of the system is like the one observed in direct optical imaging (Figs. 1 and 3), including the faint extensions (just above the noise in IR) suggestive of tidal interaction.

The measured magnitudes (objects are not catalogued) for the two main bodies are (i) PB 9494: $J=15.9, H=16.8$, $K=17.2$ and (ii) PB 9493: $J=16.7, H=17.5, K=17.8$. Within uncertainties, the $B-J$ colours of both components are thus similar and comparable to those of other IRAS galaxies. 


\subsection{Optical properties: a starburst system}

First we note that PB 9493 and PB 9494 are bright optical objects: $M_{V}=-21.3$ and $-20.3\left(h_{50}=1\right)$ compared to their linear diameters $\left(\sim 10 h_{50}^{-1} \mathrm{kpc}\right)$.

The projected separation between the luminosity peaks of PB 9493 and PB 9494 is $11 h_{50}^{-1} \mathrm{kpc}\left(13.3^{\prime \prime}\right)$ and their true relative radial velocity is small: $60 \pm 20 \mathrm{~km} \mathrm{~s}^{-1}$ for the two galaxies (or $90 \pm 20 \mathrm{~km} \mathrm{~s}^{-1}$ for their nuclei).

The continuum and emission light distributions are different: The continuum image is smooth and fuzzy but the emission lines are located mainly in the nuclei and the main bridge; conversely, the antennae are seen mostly in continuum. Those aspects are consistent with a tidal interaction generating starbursts in the nuclei and bridge as well as an ejection of antennæ made of classical stellar population.

The integrated fluxes of the PB 9494 nucleus are not exactly the same when measured from the different IFS data. This is interpreted as due to a slight offset of the telescope between the exposures: the percentage of flux lost in the interstitial space between the fibres depends on the centering. This reveals the very peaked photometric profile of the nucleus: probably less than $1.4^{\prime \prime}$ or $1.2 h_{50}^{-1} \mathrm{kpc}(F W H M)$. Line intensities, integrated on different parts of the system, have been summarized in Table 2. Due to the high intensities of emission lines, these ratios cannot significantly be affected by absorption lines of the underlying stellar components.

In the $[\mathrm{O} \text { III }]_{5007} / \mathrm{H}_{\beta}$ versus $[\mathrm{N} \mathrm{III}]_{6583} / \mathrm{H}_{\alpha}$ diagram of Veilleux and Osterbrock (1987), the two galaxies and their nuclei clearly fall in the region of HII (or starburst) galaxies. More precisely, the PB 9493-9494 system is close to the transition between classical HII galaxies and so-called "starburst nucleus galaxies".

The locations in the $[\mathrm{O} \text { III }]_{5007} / \mathrm{H}_{\beta}$ versus $\left[\mathrm{N} \mathrm{III}_{6583} / \mathrm{H}_{\alpha}\right.$ diagram of Evans \& Dopita (1985) would agree with a model of photoionization by hot stars with effective temperature $T_{*} \sim 43000 \mathrm{~K}\left(\mathrm{O} 5-\mathrm{O} 6, \sim 50 M_{\odot}\right.$ and $4 \times 10^{5} L_{\odot}$ for MS stars). This is confirmed by the diagrams $[\mathrm{O} \mathrm{III}]_{5007} / \mathrm{H}_{\beta}$ versus [S II $]_{6717+6731} / \mathrm{H}_{\alpha}$ (Veilleux \& Osterbrock 1987).

Assuming a filling factor of $10^{-3}$ and an IMF in agreement with the observed $T_{*}$ (Lequeux et al. 1981), this corresponds to a total mass of ionizing O-B stars $\simeq 5 \times 10^{7} M_{\odot}$. With $(M / L)_{V}=$ 1 (typical of starburst galaxies), this is $\simeq 10^{-3}$ of the total mass of the system and probably also a small fraction of its total gas content.

We deduce from our IFS measurements a total - and extinction corrected $-\mathrm{H}_{\alpha}$ luminosity $L_{\mathrm{H}_{\alpha}}=1.8 \times 10^{35} h_{50}^{-2} \mathrm{~W}=$ $4.8 \times 10^{8} h_{50}^{-2} L_{\odot}$. The relation of Kennicut (1983) for massive (>10 $M_{\odot}$ ) stars gives a Star Formation Rate of $2.5 M_{\odot} \mathrm{yr}^{-1}$.

Nevertheless, depending on the duration of the burst, its evolution and the shape of the IMF, the total mass of gas converted into new stars could be a factor 10-30 larger than estimated here. The fairly high concentration of the burst in the central region of PB 9494 suggests that it has evolved over at least $10^{8}$ years.

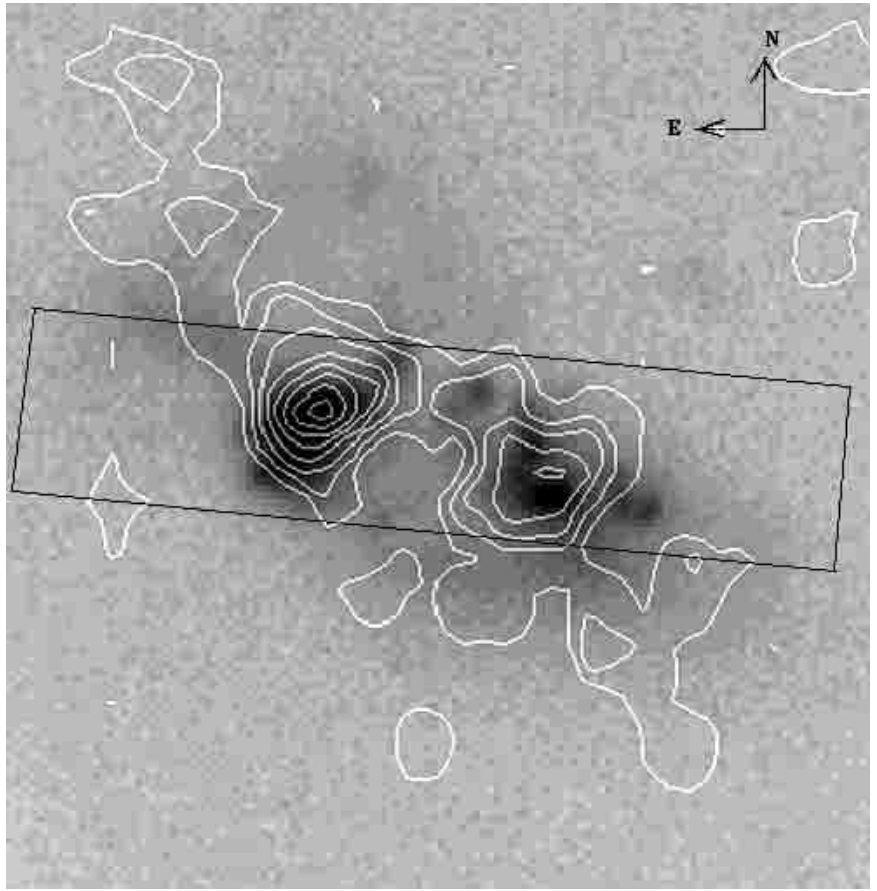

Fig. 5. $R$ and $H$ picture of PB 9493-9494 Same $R$-picture than for Fig. 1. The white superimposed isophotes are from a near-IR ( $H$ band) image available in 2MASS archives.

\subsection{The "borderline" status of PB 9494 nucleus}

The diagnostic line diagrams displaying $[\mathrm{O} \text { III }]_{5007} / \mathrm{H}_{\beta}$ versus $\left[\mathrm{N}_{\mathrm{II}}\right]_{6583} / \mathrm{H}_{\alpha}$ or versus $\left[\mathrm{S}_{\mathrm{II}}\right]_{6717+6731} / \mathrm{H}_{\alpha}$ are widely used because the involved lines are commonly strong. But the $\left[\mathrm{O}_{\text {III }}\right]_{5007} / \mathrm{H}_{\beta}$ versus $[\mathrm{O}]_{6300} / \mathrm{H}_{\alpha}$ test is a much better discriminant for the characterization of the central source essentially because the $\left[\mathrm{OI}_{6300}\right.$ line is a quite unbiased measure of collisional excitation by hot free electrons: those hot electrons are produced by photoionizing $X$ rays penetrating the wide zone of partly ionized gas around a source emitting a power-law spectrum (AGN) and are then much scarcer when the continuum is the classic thermal emission of hot stars.

We note that in this $\left[\mathrm{O}_{\mathrm{III}}\right]_{5007} / \mathrm{H}_{\beta}-[\mathrm{O} \mathrm{I}]_{6300} / \mathrm{H}_{\alpha}$ diagram PB 9494 falls in the region of Ultra Luminous Infra Red galaxies (Sanders 1992). But the main point is the discriminating power of that diagram between HII and AGNs (Veilleux \& Osterbrock 1987; Agüero et al. 1994). As seen in Fig. 6 the situation is ambiguous: if the spectrum integrated over the main body of the galaxy PB 9494 can still suggest a starburst, the one of the PB 9494 nucleus clearly enters the region of AGNs with an excitation dominated by a power-law spectrum.

Using the " $J$ " magnitudes of Vader \& Simon (1987) as approximate $B$ magnitudes and a distance modulus $36.3\left(H_{\mathrm{o}}=\right.$ $50, q_{\mathrm{o}}=0.5$ ), we deduce $B$ luminosities $L_{B}=2.5 \times 10^{10}$ and $1.2 \times 10^{10} L_{B \odot}\left(M_{B} \approx-20.7\right.$ and -19.9$)$. The $L_{\mathrm{FIR}} / L_{B}$ ratio is then 1.2 (if the FIR flux is emitted by the two objects) or 1.8 (if PB 9494 only is emitting, which is more likely). We can plot the system in the $L_{\mathrm{IR}}$ vs. $L_{B}$ and $L_{\mathrm{H}_{\alpha}}$ vs. $L_{\mathrm{IR}}$ diagrams (Coziol 1996). In the latter diagram the system is located at the frontier between HII galaxies and SBNG. In the former it lies between 


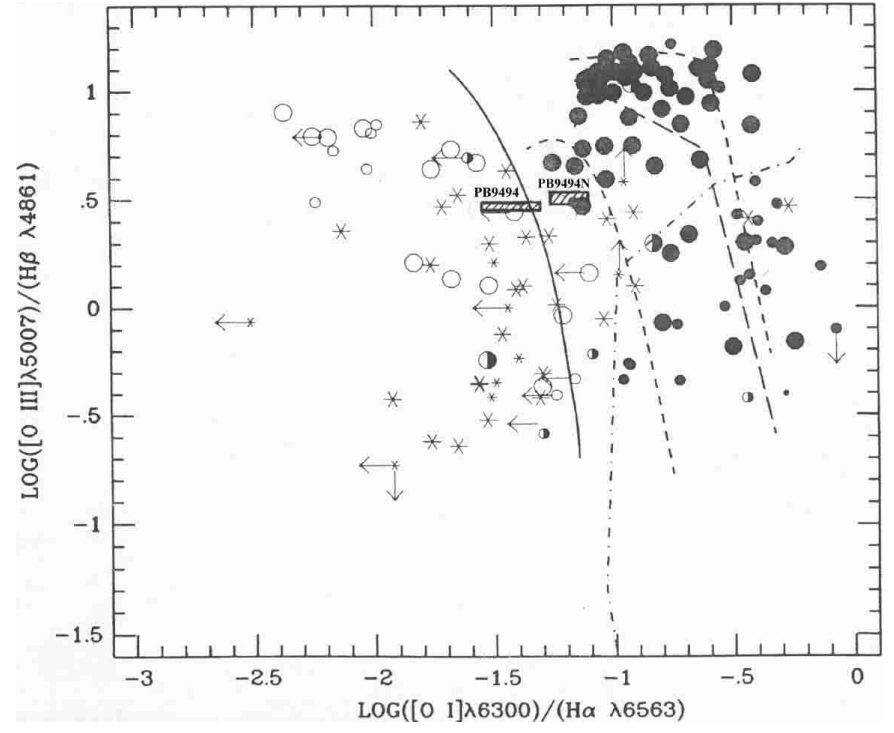

Fig. 6. Reddening-corrected $\left[\mathrm{O}_{\mathrm{III}}\right]_{5007} / \mathrm{H}_{\beta}$ versus $\left[\mathrm{O}_{\mathrm{I}}\right]_{6300} / \mathrm{H}_{\alpha}$ intensity ratios. On this copy of Fig. 6 of Veilleux and Osterbrock (1987), the "solid curve divides AGNs [filled circles] from HII region-like objects [open cicles]". We added the error boxes (hatched rectangles) of our dereddened measures of "PB9494" (whole galaxy) and "PB9494N" (its nuclear region) to this diagram.

IRAS Starbursts and Seyfert 2, very far from optical LINERs but at the frontier of IRAS LINERs.

The complexity of the morphology as well as velocity field and the small redshift difference between the 2 galaxies (which remain clearly identifiable entities) suggest that the system will evolve toward complete merging. The PB 9493-PB 9494 system is thus an example of interaction between 2 galaxies in which a general starburst is produced, with the peculiarity that one of the nuclei seems to be in the process of becoming an AGN. This may be surprising at such an early stage of a merging.

\section{Conclusions}

The correlation of IRAS sources with close pairs of PB UVexcess objects essentially supplies one clear pair of "interactivating" galaxies: the PB 9493-9494 system. This object appears quite special. Its main characteristics may be summarized as follows:

1. Our optical data show a relatively close (projected separation $\sim$ diameter) encounter of bright galaxies of similar sizes.

2. This interacting pair is dynamically evolved. This is shown by the intricacy of both its morphology and its velocity field, and by the likely stellar population of the antennæ.

3. The system has a warm FIR colour temperature, a mid FIR luminosity.

4. When comparing its optical and FIR properties, the system seems quite atypical with optical aspects of more luminous (and evolved) FIR galaxies.

5. The line ratios confirm a border-line position between several classes of active galaxies, with the nuclear region of
PB 9494 entering the AGN domain. The peculiar state of evolution for that interactive galaxy encounter could result from the peculiarities of its selection criteria (binarity, UVe and IRe).

6. Its mixed characteristics (hot dust but mid FIR luminosity, strong emission-lines and noticeable extinction) reveal an interactivated system that would display its optically luminous starburst but is still in a less evolved (dusty) state than standard IRAS encounters.

The current scenario for AGN formation involves galaxy interaction that first produces a strong starburst; then the circumnuclear starburst evolves into AGN (see e.g. Scoville 1990). Observational evidence is scarce, however. Maybe because of its selection criteria, PB 9493-PB 9494 could be a rare example of object "caught in the act", i.e. at the very early step of transition from a starburst system into an AGN.

As such, it deserves further observations with more powerful means.

We also suggest that it would be interesting for statistical studies to extend this method of selection to larger and deeper UV and IR surveys that are now available or in progress. One may think for instance, of the SLOAN and ISO databases

Acknowledgements. We are particularly grateful to A. M. Fringant who kindly drew our attention to these objects. We are indebted to P. Magain who took preliminary CCD picture of the system for us.

To Christian: Christian Vanderriest suddenly died in February 2002 during the submission process. He is the main author of this paper. The order of the names is only the result of our policy to alternate them in each paper we published together. I will remain indebted to him for that and in many other respects. (Henri Reboul)

\section{References}

Agüero, E., Calderón, J., Paolontonio, S., \& Suárez-Boedo, E. 1994, PASP, 106, 978

Bahcall, J., Kirkhakos, S., Saxe, D., \& Schneider, P. 1997, ApJ, 479, 642

Balzano, V. 1983, ApJ, 262, 602

Berger, J., \& Fringant, A. M. 1977, A\&AS, 28, 123

Berger, J., \& Fringant, A. M. 1980, A\&AS, 39, 39

Berger, J., \& Fringant, A. M. 1984, A\&AS, 58, 565

Berger, J., Cordoni, J.-P., Fringant, A.-M., et al. 1991, A\&AS, 87, 389

Burstein, D., \& Heiles, C. 1982, AJ, 87, 1165

Coziol, R. 1996, A\&A, 309, 345

Crampton, D., Cowley, A., Hickson, P., et al. 1988, ApJ, 330, 184

Djorgovski, S., Perley, R., Meylan, G., \& McCarthy, P. 1987, ApJ, 321, L. 17

Evans, I., \& Dopita, M. 1985, ApJS, 58, 125

Halpern, J., Marshall, H., \& Oke, J. 1984, AJ, 89, 1802

Keel, W. 1985, AJ, 90, 2207

Kennicut, R. 1983, ApJ, 272, 54

Lequeux, J., Maucherat-Joubert, M., Deharveng, J.-M., \& Kunth, D. 1981, A\&A, 103, 305

Lonsdale, C., Helou, G., Good, J., \& Rice, W. 1985, in Catalogued galaxies and quasars observed in the IRAS Survey (Pasadena, Jet Propulsion Laboratory)

Mac Alpine, G., Lewis, D., \& Smith, B. 1977, ApJS, 35, 203 
Mazzarella, J., Bothun, G., \& Boroson, T. 1991, AJ, 101, 2034

Miller, J., \& Mathews, W. 1972, ApJ, 172, 593

Moreau, O., \& Reboul, H. 1995, A\&AS, 111, 169

Murphy, T., Armus L., Matthews, K., et al. 1996, AJ, 111, 1025

Oke, J. 1974, AJS, 27, 21

Reboul, H., \& Cordoni, J.-P. 2002, in preparation

Reboul, H., Fringant, A.-M., \& Vanderriest, C. 1985, in Proc. of the IAU Symp. 119, Bangalore (D. Reidel Pub.), 547

Reboul, H., Vanderriest, C., Fringant, A.-M., \& Cayrel, R. 1987, A\&A, 177, 337

Sanders, D. 1992, in Relationships between active galactic nuclei and starburst galaxies, ed. A. Fillipenko, ASP Conf. Ser., 31, 303

Sanders, D., Soifer, B., Elias, J., et al. 1988, ApJ, 325, 74

Salzer, J., \& MacAlpine, G. 1988, AJ, 96, 1192

Scoville, N. 1990, in Proceedings Kona Symposium on Submillimiter Astronomy, ed. G. Wat, \& S. Webster, Astroph. and Space Sc. library, 158, 197
Sekiguchi, K. 1987, ApJ, 316, 145

Shaver, P., \& Chen, J.-S. 1985, A\&A, 148, 443

Stockton, A., \& MacKenty, J. 1987, ApJ, 316, 584

Terlevich, R., Melnick, J., Masegosa, J., Moles, M., \& Copetti, M. A\&AS, 91, 285

Thomson, D., \& Djorgovski, S. 1990, PASP, 102, 959

Vader, J. P., \& Simon, M. 1987, AJ, 94, 854

Vanderriest, C. 1995, ASP Conf. Ser., 71, 209

Vanderriest, C., \& Lemonnier, J. P. 1988, in Instrumentation for ground-based optical Astronomy, Proc. IXth Santa Cruz workshop, 304

Vanderriest, C., \& Reboul, H. 1991, A\&A, 251, 43

Veilleux, S., \& Osterbrock, D. 1987, ApJS, 63, 295

Wang, Y., \& Biermann, P. 2000, A\&A, 356, 808

Whitford, A. 1958, AJ, 63, 201 